The ITER Outline Design has been improved from the standpoints of reliabilty, safety and ease of manufacturing and assembly.

\title{
Working Towards the ITER Interim Design
}

\section{G. Janeschitz from the ITER-JCT Joint Work Site, Garching, presented the status of ITER on behalf of the ITER-JCT and Home Teams at the 22nd European Conference on Controlled Fusion and Plasma Physics.}

After the ITER Design Assessment conducted by the Joint Central Team (JCT) with the Home Teams in October 1994, a joint effort was started to simplify assembly, maintenance and manufacturing and to reduce costs while maintaining the plasma performance of the July 1994 ITER Outline Design and remaining within the overall cost expressed in 1989 ITER accounting units. Magnet coils representing $\approx 40 \%$ of the total cost of ITER received particular attention. The design improvements retain key performance parameters such as burn time, fusion power and neutron loading while reducing somewhat margins for ignition and the burn time.

In the ITER Outline Design (OD), to maximise the plasma cross-section for a given machine size, the toroidal field coils consisted of thin, jacketed conductors embedded in shear plates. To increase stiffness, torsion was taken up by a cylindrical structure through shear keys. But there were disadvantages, notably difficult maintenance of the central solenoid (CS) owing to the small gap with the toroidal field (TF) coils, the large weight of the CS assembly (3000 t) requiring a special crane, and costly manufacturing of the TF coils. A new stiffer and simpler coil design includes thin, jacketed conductors embedded in grooved shear plates enclosed inside a strong steel case. A vacuum vessel in stainless steel rather than a Ni-based superalloy is recommended in order to ease licensing. The number of TF coils is 20 instead of 24, allowing fewer divertors for easier maintenance.

The new design has the same overall machine size as the $O D$, but a slightly reduced plasma minor radius (3.0 versus 2.8 $\mathrm{m})$. To compensate for the lost plasma volume and cross-section, and to keep the field ripple at the separatrix below $2 \%$, the plasma elongation is increased ( 1.55 versus 1.6). This causes an increase in growth rate of the vertical plasma instability which in turn requires a higher installed power for the poloidal field system. The smaller plasma cross-section together with the requirement that the magnetohydrodynamic safety factor $q_{95}$ (which describes the number of toroidal turns of the field lines per poloidal turn) remains $>3$ calls for a reduction of the nominal plasma current ( 24 versus $21 \mathrm{MA}$ ).

The new design calls for further experimental work in several areas, notably scaling, equilibrium impurity levels and density and pressure limits. The scaling law for constraining the modelling of ITER's plasma fits existing data for all divertor experiments, but experimental scatter implies a $25 \%$ uncertainty for ITER. The helium density in ITER's plasma is determined almost entirely by the pumping efficiency in the divertor region and not by transport in the plasma core (some 200 $\mathrm{m}^{3} / \mathrm{s}$ of pumping exhausts the $2 \mathrm{~Pa} \mathrm{~m} 3 / \mathrm{s}$ of $\mathrm{He}$ which is produced). However, the D-T gas throughput in the pumping system is determined by the $\mathrm{He}$ concentration in the gas inside the pump ducts. Experiments show that the ratio of the $\mathrm{He}$ concentration in the divertor to the central $\mathrm{He}$ concentration should equal 0.2 , equivalent to a D-T throughput of $50 \mathrm{~Pa} \mathrm{~m}^{3} / \mathrm{s}$ requiring $0.17 \mathrm{~Pa}$ in the divertor. Divertor pressures of 0.3-8 $\mathrm{Pa}$ have been observed, albeit for relatively open systems. So there is promise that the required $\mathrm{He}$ exhaust rate can be achieved in ITER.

The power limit for a high-level of confinement (H-mode plasma) appears to be related to the power through the innermost closed flux surface. Recent data confirm the linear dependence of the threshold on toroidal field, but reveal deviations from a linear density dependence. The presence in all but one of these machines of a minimum in the threshold power at densities of about $3 \times 10^{19} \mathrm{~m}^{-3}$ can influence the deduced threshold scaling. Moreover, if the power flowing across the separatrix is corrected for radiation losses, which are themselves density dependent, the scaling is changed again. The two dimensionally correct scalings for ITER give $\mathrm{H}$-mode power thresholds between $150 \mathrm{MW}$ and $300 \mathrm{MW}$ for densities of $0.5 \times 10^{20} \mathrm{~m}^{-3}$ to $1.0 \times 10^{20} \mathrm{~m}^{-3}$ in the case of a linear density scaling, and significantly smaller values for a weaker dependence. These uncertainties may indicate that the line average density is not the right scaling parameter.

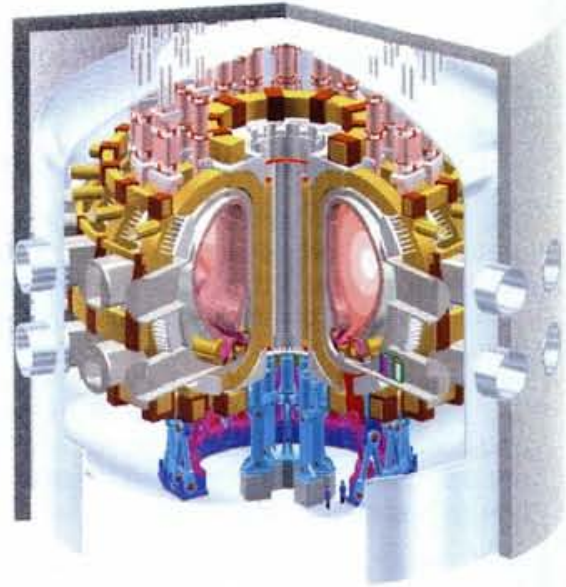

The maximum normalised plasma pressure $\beta$ permissible in ITER (the $\beta$-limit) is taken to be 2.5 at $q_{95}=3$. Experimentally, it is observed that for discharges similar to ITER's, the effective stability limit is 2.0-2.8 at $q_{95}$, indicating that the ITER value may be marginal. Some recent data show that $\beta \approx 2.7$ can be achieved at $q_{95}>3$, while at $q_{95}<3$ the $\beta$-limit drops very quickly towards about 2.5. ITER operation at $q_{95}<3$ should therefore be avoided since it would be close to the $\beta$-limit, increasing the likelihood of disruptions. However, the data are preliminary and show considerable spread so further work is needed. Reasons why the observed performance falls below the ideal MHD limit ( $\beta$ ideal $>3$ for ITER) at low $q$ are being investigated.

Increasing the auxiliary heating from 50 MW to $100 \mathrm{MW}$ will provide a reserve permitting long-pulse operation, even for energy confinement lower or equilibrium impurity levels higher than expected. Even if the fusion power has to be reduced because of lower density or $\beta$-limits than now assumed, longpulse operation at a high ratio of fusion power to auxiliary power is still possible.

\section{US Fusion Programme in Transition}

The US fusion programme is presently undergoing a transition forced by the intent of Congress to reduce the budget deficit. It is likely to experience a significant funding reduction next year. In the midst of this upheaval, the President's Committee of Advisors on Science and Technology (PCAST), through its fusion panel, completed a review and delivered a report to President Clinton's Science Advisor last July.

The PCAST report affirmed the importance of fusion energy research for the development of an attractive, and possibly essential new energy source, as well as for the value of plasma physics itself, which generates numerous insights and techniques applicable in other fields of science and technology. Based on the importance of fusion energy and the impressive scientific progress, the Committee believes that there is a strong case for the funding levels which had been envisioned by the Department of Energy (DoE), increasing from 366 M\$US in 1995 to about 860 M\$US in 2002. Although the DoE plan was viewed as reasonable and desirable, it was clear that the present budgetary constraints are unlikely to permit its realization. Hence, PCAST constructed a budget-constrained recommendation which retained what it viewed as the most indispensable elements of the US fusion programme. This $\approx 320$ M\$US p.a. plan, would inevitably sacrifice many key features of the programme as presently envisioned.

The key priorities around which the budgetconstrained strategy are organized are:

- A strong domestic core programme in plasma science and fusion technology, with funds to explore both advanced tokamak research and research on alternative concepts, leveraged where possible on related activities worldwide;

- A collaboratively funded international fusion experiment focused on the key next-step issue of ignition and moderately sustained $(\approx 100 \mathrm{~s})$ burn, costing about one-third of ITER as currently planned;

- An international programme to develop practical, low-activation fusion-reactor materials needed for economical reactor performance and environmental attractiveness.

These recommendations imply three changes from the pre-existing DoE plans. 1) That the US open negotiations with its international partners to alter the mission of ITER from one of steady-state ignition and technology to one of ignition with moderate sustainment. This recommendation followed from the fiscal limitations of the US contribution and the judgment that funding is best utilized to investigate ignition physics, with postponement of essential reactor technology development. This recommendation is made with the 